\title{
A new meta-structure of mental disorders: a helpful step into the future or a harmful step back to the past?
}

\author{
A commentary on 'A proposal for a meta-structure for DSM-V and ICD-11'
}

\author{
H.-U. Wittchen*, K. Beesdo and A. T. Gloster \\ Institute of Clinical Psychology and Psychotherapy, Technische Universitaet Dresden, Germany
}

Received 11 June 2009; Revised 10 August 2009; Accepted 15 August 2009; First published online 1 October 2009

Key words: Classification, mental disorders, psychopathology, structure.

\section{Claims and achievements of the meta-structure proposal}

The authors of the seven meta-structure papers in this issue have to be applauded for their inspiring attempt to suggest and elaborate a new meta-structure of mental disorders consisting of five 'clusters'. At first sight this proposal seems to be considerably simpler than the current diagnostic classifications structure used by DSM-IV-TR (17 categories; APA, 2000) and, in some parts, more convergent with the ICD-10 (10 categories; WHO, 1993). Against the background of dissatisfaction expressed with the current diagnostic classification structure for mental disorders and its principles, the authors provide a selective critical review of relevant research. In particular, evidence is examined to address the question of whether current individual mental disorders differ sufficiently from each other and whether the current more fine-graded distinction of specific mental disorders and their grouping in 10 major classes according to ICD-10 and 17 in DSM-IV-TR is justified. To help answer this question, a priori criteria were chosen in the form of a wide range of 'validators' grouped into so-called 'causal' risk factors (i.e. shared genetic risk factors, familiality, shared environmental risks, shared neural substrates, shared biomarkers, shared temperamental antecedents) and factors thought to be more likely to reflect the clinical picture itself (shared abnormalities of cognitive and emotional processing, symptom similarity, rates of co-morbidity, course, treatment response). The results of this impressive exploration are interpreted to suggest a substantial reduction of the current major classes to five clusters and a fairly

\footnotetext{
* Address for correspondence: H.-U. Wittchen, Ph.D., Institute of Clinical Psychology and Psychotherapy, Technische Universitaet Dresden, Dresden, Chemnitzer Str. 46, Germany.

(Email: wittchen@psychologie.tu-dresden.de)
}

large residual category of disorders not yet assigned. Particularly noteworthy examples for classificatory changes associated with the proposed meta-structure involve: the suggestion to group anxiety, somatoform and depressive disorders together under the term 'emotional disorders'; the allocation of bipolar disorders to the 'psychotic cluster' ; and the formation of a broad externalizing cluster that comprises substance use disorders, some of the personality disorders and impulse control disorders.

Several core motivations are presented by the authors to justify their proposal. (1) Increased utility, to the degree that the current system has been found to be overly complicated and too complex for routine care, in particular by non-specialists such as primary care physicians. From this perspective, the proposed meta-structure is also expected to provide considerable simplification for research and a better adoption in routine care and public administration. (2) Increased validity and advantages for research, in the sense that the proposed grouping would move classification of mental disorders away from one based purely on symptomatology and closer to the current understanding of shared putative causal risk and clinical factors identified in research. (3) Increased homogeneity within clusters, in that the proposed meta-structure would more appropriately highlight similarities rather than subtle differences between our current specific diagnoses.

Each article reviews the supporting evidence across a wide range of areas stemming from the 11 predetermined validating criteria (see Andrews et al. $2009 a$ ). These 11 criteria are a more fine-graded extension of the five original core criteria suggested by Robins \& Guze (1970) for establishing the validity of a diagnosis. Aiming for a less descriptive and more aetiological classification structure, the authors claim that particular emphasis was given to those factors 
thought to be 'causal' risk factors (e.g. shared genetic risk factors and shared neural substrates).

Taking into account the many existing diagnoses of mental disorders and the extremely rich and often fragmented research studies, undoubtedly each paper does a very impressive job of not only reviewing the available evidence but also carefully weighing the pros and cons of including and excluding disorders and the designation of disorders to clusters. However, the exposition and the proposed metastructure also raises many questions and concerns and leaves the author of this commentary puzzled in many ways.

\section{Primary appraisal: back to the 1950s - little new?}

Three immediate and puzzling impressions arise upon initial reflection of the proposed meta-structure when it is compared to current and past ICDs and DSMIV-TR. The first is that we have stepped into the past. As displayed in Table 1, the very broad 'emotional cluster', which lumps all depressive, anxiety, stressrelated and somatoform disorders and also avoidant personality disorder together, and the 'psychosis cluster', which includes bipolar and schizotypical personality disorder, resemble the neurotic-psychotic distinction in ICD-6 (psychoses and psychoneurotic disorders). Although largely consistent with the current two-digit ICD-10 codes, this grouping not only represents a substantial change from the current DSM but also seems to be counterintuitive given the extremely rich research literature on specific 'emotional' disorders and their differences with regard to at least some of the 'validators'.

Similarly, the lumping of substance use disorders, under the broad umbrella of externalizing disorders, together with conduct, antisocial and borderline personality disorders and also impulse control disorders is astonishing and resembles - with the exception of intelligence - the ICD-6 grouping of 'Disorders of character, behavior and intelligence'. After decades of multifaceted substance use research based on highly differentiated classification of substance use disorders by substance class, short- and long-term complications, and the core phenomenon of addiction, one is hard-pressed to accept the premise of the metastructure, that all substance use disorders could be appropriately grouped under the term 'externalizing'. Is there really no evidence for a separate substance use cluster? Or could it be that the proposition is based only on a particular set of literature limited to substance dependence?

The second impression is that, beyond the emotional and externalizing clusters, there seems to be little new. This impression arises despite the fact that, at the molecular level, the grouping of disorders is slightly different, as are the justifications. What was previously 'Disorders Usually First Diagnosed in Infancy or Adolescence' in DSM has now become the 'Neurodevelopmental cluster'; 'Delirium, Dementia, Amnestic and Other Cognitive Disorders' are now labelled the 'Neurocognitive cluster'; and 'Schizophrenia and psychotic disorders' are now the 'Psychotic cluster'. However, it should be noted that, across all clusters, some core diagnoses and wellestablished major groups that would intuitively be seen as separate clusters are in fact subsumed under one new unusual label (e.g. mood and anxiety or somatoform disorders under emotional) or moved to other clusters (e.g. bipolar disorders to psychotic). Although there seem to be some good reasons for their grouping, some of these changes are not intuitively clear and might give rise to some confusion.

A third impression is that the logic and procedure chosen by the meta-structure group leaves many DSM disorders unclassified (see Table 1). This occurs either for conceptual reasons (e.g. departure of DSM-IV-TR distinction of Axis I and Axis II disorders, and restriction to 'primary mental disorders' disregarding mental disorders due to a general medical condition), because of a lack of research data (e.g. elimination disorders, tics, some personality disorders, body dysmorphic, factitious, dissociative disorders, sleep disorders, sexual and gender identity disorders), or for unknown reasons (e.g. the majority of substance userelated disorders, except substance dependence). The large proportion of disorders not yet assigned raises a range of immediate concerns: Will those currently omitted ultimately fit one of the proposed clusters? Will further clusters be derived? Will some rare disorders ultimately form new clusters based on separate evidence and principles than those assigned already to the five clusters?

Beyond these general and immediate impressions, our initial enthusiasm was further reduced when considering the numerous limitations, some of which are acknowledged in the introductory paper (Andrews et al. 2009a). Core examples of the limitations include the top-down approach, which seems to be strongly and disproportionately driven by studies that have examined the structure of mental disorders based on co-morbidity using higher-order factor analysis, and the entire lack of evidence that the proposed clusters will be useful in research and practice. These concerns prompt the question of whether the proposed metastructure is indeed a step forward to an improved classification structure that could be described as 'a more parsimonious meta-organization ... (of the mental disorders emphasizing) ... risk factors, increasing clinical utility and potentiate research into the 
Table 1. The classification of mental disorders from ICD-6 (1948) to the proposed meta-structure clusters

\begin{tabular}{|c|c|c|c|}
\hline ICD-6 (WHO, 1948) & $\begin{array}{l}\text { ICD-10 Mental and behavioural disorders } \\
\text { (F00-F99) }\end{array}$ & DSM-IV-TR & $\begin{array}{l}\text { Proposed meta-structure and } \\
\text { relationship to DSM-IV-TR }\end{array}$ \\
\hline $\begin{array}{l}\text { (300-309) Psychoses } \\
300 \text { Schizophrenic disorders }\end{array}$ & $\begin{array}{l}\text { F00-F09: Organic, including symptomatic, } \\
\text { mental disorders }\end{array}$ & $\begin{array}{l}\text { Disorders usually first diagnosed in } \\
\text { infancy or adolescence }\end{array}$ & Cluster 2: Neurodevelopmental disorders \\
\hline $\begin{array}{l}301 \text { Manic-depressive reaction } \\
302 \text { Involutional melancholia }\end{array}$ & $\begin{array}{l}\text { F10-F19: Mental and behavioural disorders } \\
\text { due to psychoactive substance use }\end{array}$ & $\begin{array}{l}\text { Delirium, dementia and amnestic and } \\
\text { other cognitive disorders }\end{array}$ & Cluster 1: Neurocognitive disorders \\
\hline $\begin{array}{l}303 \text { Paranoia and paranoid states } \\
304 \text { Senile psychosis } \\
305 \text { Presenile psychosis }\end{array}$ & $\begin{array}{l}\text { F20-F29: Schizophrenia, schizotypal and } \\
\text { delusional disorders } \\
\text { F30-F39: Mood [affective] disorders }\end{array}$ & $\begin{array}{l}\text { Mental disorders due to a general } \\
\text { medical condition not elsewhere } \\
\text { classified }\end{array}$ & Not considered and assigned \\
\hline $\begin{array}{l}306 \text { Psychosis with cerebral arteriosclerosis } \\
307 \text { Alcoholic psychosis } \\
309 \text { Other and unspecified psychoses }\end{array}$ & \multirow{3}{*}{$\begin{array}{l}\text { F40-F48: Neurotic, stress-related and } \\
\text { somatoform disorders } \\
\text { F50-F59: Behavioural syndromes associated } \\
\text { with physiological disturbances and } \\
\text { physical factors }\end{array}$} & $\begin{array}{l}\text { Schizophrenia and other psychotic } \\
\text { disorders }\end{array}$ & $\begin{array}{l}\text { Cluster 5: Externalizing disorders } \\
\text { Cluster 3: The psychoses }\end{array}$ \\
\hline (310-318) Psychoneurotic disorders & & Mood disorders & Cluster 4: Emotional disorders \\
\hline 310 Anxiety reaction w.o. somatic sxs & & Anxiety disorders & Cluster 4: Emotional disorders \\
\hline 311 Hysterical reaction w.o. anxiety reaction & $\begin{array}{l}\text { F60-F69: Disorders of adult personality and } \\
\text { behaviour }\end{array}$ & Somatoform disorders & Cluster 4: Emotional disorders \\
\hline 312 Phobic reaction & & $\begin{array}{l}\text { Factitlous disorders } \\
\text { Dissociative disorders }\end{array}$ & $\begin{array}{l}\text { Not assigned } \\
\text { Not assigned }\end{array}$ \\
\hline 314 Neurotic-depressive reaction & \multirow{4}{*}{$\begin{array}{l}\text { F80-F89: Disorders of psychological } \\
\text { development } \\
\text { F90-F98: Behavioural and emotional } \\
\text { disorders with onset in childhood } \\
\text { and adolescence }\end{array}$} & Sexual and gender identity disorders & Not assigned \\
\hline 31X Psychoneurosis with somatic sxs & & Eating disorders & Not assigned \\
\hline $315 \ldots$ affecting circulatory system & & Sleep disorders & Not assigned \\
\hline $\begin{array}{l}316 \ldots \text { affecting digestive system } \\
317 \ldots \text { affecting other systems }\end{array}$ & & $\begin{array}{l}\text { Impulse-control disorders not elsewhere } \\
\text { classified }\end{array}$ & Cluster 5: Externalizing disorders \\
\hline $318 \ldots$ other, mixed and unspecified types & \multirow[t]{9}{*}{ F99: Unspecified mental disorder } & Adjustment disorders & Cluster 4: Emotional disorders?? \\
\hline $\begin{array}{l}\text { (320-326) Disorders of character, } \\
\text { behaviour and intelligence }\end{array}$ & & \multirow{8}{*}{$\begin{array}{l}\text { Other conditions that may be a focus of } \\
\text { clinical attention }\end{array}$} & \multirow[t]{8}{*}{ Not considered and assigned } \\
\hline $\begin{array}{l}320 \text { Pathological personality (e.g. antisocial } \\
\text { personality) }\end{array}$ & & & \\
\hline $\begin{array}{l}321 \text { Immature personality (e.g. emotional } \\
\text { instability) }\end{array}$ & & & \\
\hline 322 Alcoholism & & & \\
\hline 323 Other drug addiction & & & \\
\hline 324 Primary childhood behaviour disorders & & & \\
\hline 325 Mental deficiency & & & \\
\hline $\begin{array}{l}326 \text { Other and unspecified character, } \\
\text { behaviour and intelligence disorders }\end{array}$ & & & \\
\hline
\end{tabular}


cause and prevention of mental disorders' (Andrews et al. 2009a). They also stimulate the alternative question: Does the proposed meta-structure harbour considerable potential for confusing and misleading researchers and clinicians?

\section{A secondary appraisal of the meta-structure: general issues}

On a more general level, we first want to consider the motivation for suggesting the meta-structure, its meaning, and its future role.

\section{Technical harmonization?}

Theoretically, revisions of a diagnostic classification system should be driven by solid scientific evidence for introducing necessary changes that improve the core functions of a classificatory system. For example, changes should improve communication in research and practice and improve utility in terms of treatment, intervention and prognosis. In reality, however, revisions are strongly driven by additional motivations, such as the drive to be different from the past, political concerns, and technical reasons. Bearing this in mind, the meaning of the proposed meta-structure is not entirely clear. Is it simply meant to simplify the grouping of the disorders by significantly reducing the number of major classes? This might indeed be a worthwhile goal if it were simply for technical/ pragmatic reasons. The alphanumeric system of ICD chapters, for example, only allows for 10 major groups and it would be desirable if there was a greater concordance with the current DSM-IV-TR classification, which currently specifies 17 . Such concordance would reduce the potential for problems between the classificatory systems. Harmonization between the two systems could simply occur by reducing the number of DSM categories by a more or less arbitrary or 'technical' procedure that combined major DSM groups; in fact the current ICD-10 provides an example, though imperfect, for this. Alternatively, a more radical approach could be used, such as the one suggested with the meta-structure proposal. If such a 'technical harmonization' is the primary intention, then the meta-structure proposal would not do any major harm because it allows individual diagnoses to be retained, albeit under different umbrellas within the meta-structures five clusters.

However, the current proposition mentions five clusters, leaving open how the remaining slots will be filled. This opens the dangerous possibility that additional clusters will be added with other groups of disorders, but without a similarly rigorous approach used to derive the five meta-structure clusters suggested in this issue. Alternatively, it is possible that there will be only one residual class. Having been involved in some of the 'political negotiations' of past DSM and ICD revisions, we assume the former will happen, resulting in a mixed bag of classificatory structures.

\section{Deeper theoretical meaning and implication?}

We struggled with the fact that the meta-structure proposition was obviously intended to have a 'deeper' meaning. All papers emphasize repeatedly that the proposition 'is a step away from a classification based on symptom picture alone' (Andrews et al. $2009 a$ ) towards 'a classification based on etiological risk factors' (Andrews et al. 2009a). Across variations in terminology, all papers emphasize the role of 'causal risk' factors or 'spectrum's phenotypic coherence' (Krueger \& South, 2009) and suggest that the findings move us towards a deeper aetiological meaning. Within this context, the authors claim that the emotional cluster disorders, for example, share 'emotional dysregulation and negative emotionality' as core defining features whereas the externalizing cluster disorders share 'disinhibition'. To the degree that this is the case, significant concerns arise.

(a) Given that the evidence presented in favour of the proposed clusters is selective and 'biased' towards the similarities rather than the differences between disorders, we question the claim that the metastructure will be helpful. How significant and substantial is the danger that the guiding principles will misguide future research or even negatively impact our clinical practice?

(b) The proposal is strongly driven by one theoretically and methodologically problematic approach, namely explorations of higher-order factor analytic methods. Based largely on studies of co-morbidity patterns in the community, these studies suggest that symptom and diagnostic data might be best grouped into an internalizing factor (i.e. panic disorder, generalized anxiety disorder, agoraphobia, specific and social phobia in addition to major depression and dysthymia) and an externalizing factor (i.e. substance dependence, conduct and antisocial personality disorders). However, the authors of the meta-structure papers should have also acknowledged the severe limitations of this methodological approach and the data upon which it is based (Wittchen et al. 1999, 2009). The most relevant limitations are the statistical method, the lack of developmental stability, and the lack of structural stability when additional disorders are considered. In fact, these factor analytic studies have been based on only some (maximum 12 disorders) of the disorders considered for the five clusters and only a minority of all DSM disorders. Therefore, 
the rationale and designation of additional disorders above and beyond the factor analytic evidence remains unclear in many sections.

(c) Alternative meta-structure approaches exclusively based on single risk factor domains (e.g. neurobiological, familiality, shared specific environmental factors), course and outcome or other perspectives such as 'staging of disorder progression' (Shear et al. 2007), or functional-behavioural or linguistic concepts (Hayes et al. 2001) were neither considered nor explored systematically. The scientific evidence presented in the meta-structure papers was always 'forced to fit' the top-down derived meta-structure emphasizing similarities and 'downplaying' differences. How heavily should we weight the danger that this strategy misses or discards important and meaningful signals for core psychopathological mechanisms and processes?

(d) In many papers, we struggled with the authors' use of the term 'causal' risk factors. The overemphasis on 'causal' risk factors occurred to the exclusion of other factors. Furthermore, the authors seem to assume that the availability of family-genetic and neurobiological evidence in many domains is tantamount to causal proof for a mental disorder. This seems to be overly optimistic. To date, for almost all disorders there is little evidence for any causal factor or mechanism that is both necessary and sufficient for a specific mental disorder and even the status of many measures as reliable 'markers' is typically controversial. Throughout the papers, the authors only poorly address the degree to which these 'neurobiological' measures are merely correlates of disturbances in cognition, emotion and behaviour inherent in the respective condition considered and across all mental disorders. Clearly, all mental disorders must be associated with disturbances in neural mechanisms but, with a few diagnostic exceptions, a core causal role as precipitant is as questionable as it is for psychological and environmental factors (see, for example, Andrews et al. 2009b). It might be more appropriate to assume that all these factors interact dynamically and might all be responsible in a probabilistic way at all times for all disorders in some way or another. This, of course, is difficult to capture in a structural appraisal of a diagnostic classification system.

(e) Some of the major limitations of the metastructure proposal are addressed in the introductory paper. These include the 'top-down' approach, lack of systematic reviews, lack of coherent and comprehensive data supporting the proposed clusters, incomplete and partly contradictory evidence in terms of the 11 criteria, largely lacking proof-of-concept information, lack of systematic exploration and testing of alternative grouping, and the partly assumed cluster membership of a diagnosis without data and research evidence. Despite these understandable but considerable limitations, the papers frequently seem to overstate what they found in many places. Claims that the data 'validate' the clusters (Andrews et al. 2009a) or that the meta-structure proposition relied on (causal) risk factors and clinical factors and is thus 'more parsimonious' (Andrews et al. 2009a) seem to go much too far.

\section{'Deeper' practical implications?}

Is the proposition meant to suggest or would its implementation result in the deletion of certain groups of disorders or even certain diagnoses from our research and clinical practice agenda? To what degree is the meta-structure meant to replace current diagnostic practice by allowing the cluster labels to be used as a sufficient global diagnostic substitute? To what degree is it meant to reduce spurious co-morbidity, by assuming that 'relevant' co-morbidity only exists between the five clusters? Although it is prominently emphasized several times that the meta-structure is not meant to delete disorders nor should the clusters be seen as mutually exclusive, inconsistent claims and suggestions for each of these aims can be found across all papers. Given that these questions are not considered systematically, nor are the potential dangers explored, we feel uncomfortable about the ultimate goal, namely the actual use of the proposed metastructure and its implications for the field. And given the very limited and exploratory evidence for the clusters, adoption of the proposed meta-structure seems premature. It is therefore disconcerting that Andrews et al. (2009a) strongly advocate a major broad-range research programme not only to explicate the neurobiological and other cluster risk factors but also to use the proposed meta-structure as a base for research on treatment, prognosis, and even practice.

In terms of increased clinical utility, there is no explanation of why the proposition would be expected to have greater utility and validity compared to the current DSM grouping. This is especially concerning in that this 'fact' is explicitly stated in several papers. For example, Andrews et al. (2009a) state that, for internists and general practitioners, 'the clustering will simplify an otherwise confusing system'. Yet the papers do not specify how and why a simplification will work, even when a specific diagnostic category still needs to be reliably ascertained before deciding on cluster membership. To be fair, the authors admit this problem indirectly in other places when they state that the utility should be tested and that primary proof of concept studies are needed. 


\section{Harmful effects?}

The authors of this proposition fail to reflect on the potentially significant negative side-effects. Leaving aside the substantial gaps with regard to supporting data, might the lumping of anxiety, somatoform and depressive disorders under the very broad umbrella of 'emotional cluster' revive the stigmatizing and otherwise controversial concept of neurosis? The initial beauty and simplicity of grouping mental disorders into conditions that could be mainly characterized by 'emotional dysregulation', as in the emotional cluster, and 'disinhibition', as in the externalizing cluster, does not protect against the revival of potentially stigmatizing diagnostic group labels. Even worse, such an organization carries an increased risk of losing sight of research-based distinctions and considerably different intervention needs of the specific anxiety, depressive, somatoform and other 'emotional cluster' disorders. It took two decades to communicate, albeit sometimes too subtly, that diagnosing specific mental disorder matters. For example, it is true that the crosssectional picture of a person with panic disorder with past uncued panic attacks might not be that different from agoraphobia, severe specific phobia, generalized anxiety disorder or even depression with anxiety features when considering presenting symptoms, distress, and associated neurobiological and behavioural correlates. However, doesn't the occurrence of initial spontaneous panic attacks tell us something extremely useful with regard to prognosis (Wittchen \& Gloster, 2009; Wittchen et al. 2009) and to treatment? Isn't there persuasive evidence that panic disorder without agoraphobia is a quite different 'animal' from panic disorder with agoraphobia (Wittchen et al. 2009)? How damaging will a broad cluster proposition be in this respect if a 'deeper' meaning is involved?

In addition, just as these authors suggest that progress is impeded by the current system's failure to use meta-structures, we must ask what progress might be impeded if these meta-structures are incomplete or incorrect. Intended or not, the diagnostic system will always influence the field's thinking about psychopathology and treatment. Is it therefore wise to adopt a meta-structure with a 'deeper meaning' that has not been adequately tested?

\section{Secondary appraisal: specific issues}

Beyond the more general issues regarding the metastructure proposition, there is also a range of important issues pertaining to some of the specific clusters and the assignment of disorders. As an example we focus on the presumably largest, namely 'the emotional' cluster. First, the label seems to be misleading. There is little evidence that emotional disturbances play a more significant role within this cluster than cognitive, behavioural and physiological factors. Furthermore, the exceedingly high overlap of anxiety and depressive and somatoform disorders with almost all other clusters (including the psychoses, the neurocognitive and externalizing cluster disorders) that may occur primarily, concurrently or secondary to all other cluster disorders makes it difficult for us to understand the justification for this broad cluster and its label. Second, the lumping together of anxiety, depressive and somatoform disorders seems to reflect misguided emphasis on cross-sectional subjective verbal phenomenological data (in this respect the term negative affect might be more appropriate), whereas the differential effects of course, developmental, neurobiological and treatment evidence are downplayed. Indeed, when examining the cited references, it seems that the differences between disorders in this cluster (e.g. abnormalities of cognitive and emotional processing, familiality and treatment) are greater than those observed between clusters. Precedence in the decision to assign these heterogeneous conditions to one cluster seems to be more driven by the common final pathway of presentation of these conditions than by the actual data of the course, treatment, familiality and cognitive and emotional processing. Similar convergent evidence could easily be found for the formation of at least three clusters, for example primary anxiety, mood and obsessive-compulsive spectrum or stress/traumarelated disorders. Third, the placement of bipolar disorder in the psychotic cluster seems to be consistent with a substantial body of evidence that some forms of mood disorders might be more closely related to the psychoses spectrum (or vice versa). Indeed, research evidence suggests that both psychotic and mood disorders can be grouped best on a continuum. However, why single out bipolar and not ' major depression with psychotic features'?

\section{Conclusions}

Will this new meta-structure make researchers and clinicians in the mental health field happier? Is the proposed meta-structure really more appropriately based on 'validators' and particularly those that are assumed to be 'causal' risk factors? Will it stimulate better research? Will it improve clinical utility and practicality in routine care? Will it improve treatment and care?

After careful consideration and re-examination of the evidence presented, we have come to the conclusion that all these questions have to be answered in the negative. Even though the meta-structure series reflects an almost 'Herculean' effort, as one of the 
authors described it, the outcome seems to be closer to 'Sisyphean'! The research evidence presented clearly signals that it is already extremely difficult to comprehensively 'validate' one single disorder. Why should it be easier or even more feasible to validate clusters of heterogeneous disorders? Given the significant limitations of the approach and the current lack of empirical support (unlikely to become available during the next few years), the proposed metastructure and its claims should be regarded as an interesting exploration but not as a solid or promising basis for the upcoming ICD-11 or DSM-V revisions.

If the intent and motivation of the authors would have been more moderate - for example focusing primarily on the technical aspect of reducing the number of major groups of mental disorder without the overly ambitious claims of a deeper meaning and increased clinical utility - the proposed meta-structure would be a helpful step. However, the proposal is not persuasive when evaluated against the initial aims of developing a more parsimonious meta-structure based on risk factors and clinical factors and with the longterm aim of improving clinical practice, administration, research and even training and education. In fact, it might even imply more risks for harm than benefits in each of the domains. For example, it may lead to an oversimplification of mental disorders, premature adoption of putative risk factors, and diminish recognition and treatment.

The proposed meta-structure, with its assumed 'deeper implications', might also divert the attention of the DSM-V and ICD-11 Task Forces from searching for alternative approaches that might match researchers' and clinicians' needs more appropriately. In this respect, there is undoubted consensus that future diagnostic classification systems should adopt and incorporate meaningful dimensions and continua, stipulate how they relate differentially to the full spectrum of diagnoses, and how this information can be incorporated in the necessarily dichotomous decisions made in diagnostic classificatory systems (Regier, 2007). Some of these dimensional additions and conceptualization might be relevant to only one disorder or a smaller group of related diagnoses. Others will cut across diagnostic categories. Similar to the 'validators' of the meta-structure, these dimensions could be conceptualized as diagnostic specifiers in terms of (family) genetic factors, neural substrates or biomarkers, temperamental antecedents, core psychopathological features, basic psychological dysfunctions of cognition and affect and also course and treatment response. It remains to be determined and debated, however, which dimensions best capture salient diagnostic and therapeutic questions from a classificatory point of view.

\section{Declaration of Interest}

None.

\section{References}

Andrews G, Goldberg DP, Krueger RF, Carpenter Jr. WT, Hyman SE, Sachdev P, Pine DS (2009a). Exploring the feasibility of a meta-structure for DSM-V and ICD-11: could it improve utility and validity? Psychological Medicine. doi:10.1017/S0033291709990250.

Andrews G, Pine DS, Hobbs MJ, Anderson TM, Sunderland M (2009b). Neurodevelopmental disorders: Cluster 2 of the proposed meta-structure for DSM-V and ICD-11. Psychological Medicine. doi:10.1017/ S0033291709990274.

APA (2000). Diagnostic and Statistical Manual of Mental Disorders, 4th edn, text revision. American Psychiatric Press: Washington, DC

Hayes SC, Barnes-Holmes D, Roche B (eds) (2001). Relational Frame Theory: A Post-Skinnerian Account of Human Language and Cognition. Kluwer Academic/Plenum Publishers: New York.

Krueger RF, South SC (2009). Externalizing disorders: Cluster 5 of the proposed meta-structure for DSM-V and ICD-11. Psychological Medicine. doi:10.1017/ S0033291709990328.

Regier DA (2007). Dimensional approaches to psychiatric classification: refining the research agenda for DSM-V: an introduction. International Journal of Methods in Psychiatric Research 16, S1-S5.

Robins E, Guze SB (1970). Establishment of diagnostic validity in psychiatric illness: its application to schizophrenia. American Journal of Psychiatry 126, 983-987.

Shear MK, Bjelland I, Beesdo K, Gloster AT, Wittchen H-U (2007). Supplementary dimensional assessment in anxiety disorders. International Journal of Methods in Psychiatric Research 16, S52-S64.

WHO (1948). ICD-6. Manual of the International Statistical Classification of Diseases, Injuries, and Causes of Death (6th revision). World Health Organization: Geneva.

WHO (1993). The ICD-10 Classification of Mental and Behavioural Disorders: Diagnostic Criteria for Research. World Health Organization: Geneva, Switzerland.

Wittchen H-U, Beesdo K, Gloster AT (2009). The position of anxiety disorders in structural models of mental disorders. Psychiatric Clinics of North America 32, $465-481$.

Wittchen H-U, Gloster AT (2009). Developments in the treatment and diagnosis of anxiety disorders [Preface]. Psychiatric Clinics of North America 32, xiii-xix.

Wittchen H-U, Höfler M, Merikangas KR (1999). Towards the identification of core psychopathological processes? Archives of General Psychiatry 56, 929-931.

Wittchen H-U, Nocon A, Beesdo K, Pine DS, Höfler M, Lieb R, Gloster AT (2008). Agoraphobia and panic: prospective-longitudinal relations suggest a rethinking of diagnostic concepts Psychotherapy and Psychosomatics 77, 147-157. 